\title{
Intermédialités
}

Histoire et théorie des arts, des lettres et des techniques

Intermediality

History and Theory of the Arts, Literature and Technologies

\section{Théâtre et intermédialité}

\section{Une rencontre tardive ["Mettre en scène / Directing", no 12 automne 2008]}

\section{Jean-Marc Larrue}

Numéro 20, supplément, automne 2012, printemps 2013

traverser

crossing

URI : https://id.erudit.org/iderudit/1023528ar

DOI : https://doi.org/10.7202/1023528ar

Aller au sommaire du numéro

Éditeur(s)

Revue intermédialités (Presses de l’Université de Montréal)

ISSN

1705-8546 (imprimé)

1920-3136 (numérique)

Découvrir la revue

Citer cet article

Larrue, J.-M. (2012). Théâtre et intermédialité : une rencontre tardive ["Mettre en scène / Directing", no 12 automne 2008]. Intermédialités / Intermediality, (20),

101-117. https://doi.org/10.7202/1023528ar 


\title{
Théâtre et intermédialité \\ Une rencontre tardive
}

\author{
Jean-Marc Larrue
}

S

i les études intermédiales, qui sont nées dans le sillon de la révolution numérique, n'ont pas vingt ans, les processus qu'elles contribuent à mettre au jour remontent bien au-delà de cette dernière vague technologique majeure, comme l'a clairement illustré Remediation: Understanding New Media, l'ouvrage-clé que Jay Davis Bolter et Richard Grusin publiaient en 2000. Selon certains chercheurs, tel Peter Boenisch, les processus intermédiaux sont présents dès l'invention de l'alphabet² et les théoriciens de l'interartialité (Walter Moser ${ }^{3}$, Claus Clüver ${ }^{4}$ ) voient dans les relations complexes et constantes entre les pratiques artistiques depuis la Renaissance des modèles probants de dynamique intermédiale. Quant aux travaux menés depuis une trentaine d'années sur cette autre révolution qu'a provoquée l'avènement de

1. Jay David Bolter et Richard Grusin, Remediation: Understanding New Media, Cambridge, MIT Press, 2000.

2. Cette thèse rejoint celle de Derrick de Kerckhove pour lequel l'origine du théâtre occidental, dans la Grèce antique, est directement attribuable au développement de l'alphabet phonétique deux ou trois siècles avant les premières Dionysies (vers le $8^{\mathrm{e}}$ siècle av. J.-C.). Voir en particulier Derrick de Kerckhove, «A Theory of Greek Tragedy », SubStance, ${ }^{\circ} 29$, mai 1981, p. 23-25. Michael Darroch (infra., p. 97) aborde cette question dans le texte qu'il consacre au son et à la voix.

3. Walter Moser, «L'interartialité: pour une archéologie de l'intermédialité », dans Marion Froger et Jürgen E. Müller (dir.), Intermédialité et socialité: histoire et géographie d'un concept, Münster, Nodus Publikationen, coll. «Film und Medien in der Diskussion", vol. 14, 2007, p. 69-92.

4. Claus Clüver, «Intermediality and Interarts Studies», dans Jens Arvidson, Mikael Askander, Jørgen Bruhn et Heidrun Führer (dir.), Changing Borders: Contemporary Positions in Intermediality, Lund, Intermedia Studies Press, 2007, p. 19-37. 
l'électricité5, ils montrent bien, par les similitudes frappantes qu'on observe dans le développement des technologies électriques et la formation des médias électriques, d'une part, et celui des technologies et médias numériques, d'autre part, que les mêmes phénomènes sont à l'œuvre 6 à cent ans d'écart.

On s'étonne que le théâtre, qui a connu de remarquables mutations grâce à l'électricité et qui vit actuellement un important renouveau attribuable à l'effet conjugué de l'éclatement des barrières disciplinaires traditionnelles et de l'invasion des nouvelles technologies (numériques) - les deux ne sont pas sans lien - n'ait pas plus tôt attiré l'attention des chercheurs intermédiaux. Pratique fertile où se croisent et se mêlent, de temps immémoriaux, les arts et les technologies, où la question du dispositif est cruciale, le théâtre se trouve lié, d'une façon ou d'une autre, à tous les grands bouleversements médiatiques qui ont marqué le monde des communications et du divertissement depuis un siècle, soit qu'il y ait contribué, soit qu'il les ait subis, soit les deux à la fois. Pourtant, Bolter et Grusin évoquent à peine le théâtre dans leur essai historique; Jürgen Müller, figure de proue de l'école allemande de l'intermédialité, n’en traite qu'accessoirement; et Walter Moser, qui a pourtant examiné les manifestations les plus diverses de l'interartialité, n'a pas inclus le théâtre dans ses observations! Ce silence s'explique sans doute en partie par l'origine disciplinaire (et les intérêts) des premiers chercheurs intermédiaux. Mais il y a davantage. En affirmant la primauté des systèmes de relations sur les objets dans la dynamique et la genèse des médias et, surtout, en plaçant au centre de leurs préoccupations la question de la matérialité des processus médiatiques, les théoriciens de

5. D'ailleurs, le Centre de recherche sur l'intermédialité de l'Université de Montréal est historiquement plus lié à la révolution électrique (fin $19^{\mathrm{e}}$ et début $20^{\mathrm{e}}$ ) qu'à la révolution numérique survenue cent ans plus tard.

6. Dès 1988, Carolyn Marvin publiait un ouvrage marquant sur la question, soulignant, elle aussi, tous ces phénomènes de transfert et l'importance du milieu dans la genèse des médias électriques. Voir Carolyn Marvin, When Old Technologies Were New: Thinking About Electric Communication in the Late Nineteenth Century, New York, Oxford University Press, 1988. Les ouvrages subséquents de Lisa Gitelman (Always Already New: Media, History and the Data of Culture, Cambridge, MIT Press, 2006), celui de Siegfried Zielinski (Deep Time of the Media: Toward an Archeology of Hearing and Seeing by Technical Means, trad. Gloria Constance, Cambridge et Londres, MIT Press, coll. «Electronic Culture: History, Theory, Practice», 2006) et le développement des Sound Studies (Jonathan Sterne, The Audible Past: Cultural Origins of Sound Reproduction, Durham, Duke University Press, 2003) participent du même mouvement d'ensemble et témoignent autant de sa vitalité que de son éclectisme. 
l'intermédialité n'ont rien fait pour s'attirer la sympathie d'un milieu qui, depuis près d'un siècle, élabore un discours identitaire fondé sur l'acteur (sa voix, son corps) et son rapport immédiat au spectateur.

\section{LE RÉFLEXE IDENTITAIRE}

Jonathan Sterne ${ }^{7}$ rappelle que les slogans-concepts de «fidélité » puis «haute fidélité » - à quoi? - qu'a forgés l'industrie des technologies de reproduction sonore $^{8}$ de la fin du $19^{\mathrm{e}}$ siècle au milieu du $20^{\mathrm{e}}$ siècle n'avaient d'autre but que de contrecarrer les accusations de «trahison» portées contre ses «dispositifs » qui interféraient entre le corps de l'artiste et l'oreille de l'auditeur-spectateur. Ces dispositifs brisaient un lien que ses défenseurs prétendaient « direct», "pur», «naturel », «unique» avec, évidemment, tout ce que cela pouvait induire de nostalgie et d'appréhension. Les technologies de reproduction sonore portaient atteinte à l'aura9 de la scène qui avait été, jusque-là, le lieu unique de consécration des «belles voix». En plus des questions de reproductivité et d'authenticité qu'elle soulevait, l'intrusion du son médiatisé sur la scène du théâtre était ainsi perçue - et présentée - sous l'angle d'une double agression par ses détracteurs qui étaient aussi les défenseurs de la scène dite «séculaire » : celle de la «nouvelle culture décadente» des amusements mécaniques contre l'«autre» culture; celle de la technologie contre l'artiste et contre l'art, du factice contre le vrai. Non seulement ce son instaurait-il une première rupture historique de la voix et du $\operatorname{corps}^{1 \circ}$, mais il lançait la pratique «contre-nature » de l'écoute acousmatique ${ }^{11}$.

7. Jonathan Sterne, The Audible Past: Cultural Origins of Sound Reproduction, p. $215 s q$.

8. Destinées soit à la transmission à distance soit à l'enregistrement, elles étaient électriques ou acoustiques.

9. Le terme rendu célèbre par l'essai retentissant de Walter Benjamin n'est évidemment pas utilisé à l'époque, mais les enjeux qu'il soulève sont les mêmes.

10. Mladen Dolar, A Voice and Nothing More, Cambridge, MIT Press, coll. «Short Circuits", 2006, p. 9-12.

11. Rappelant les fameuses leçons que Pythagore donnait à ses disciples, séparé d'eux par un rideau, de sorte qu'ils l'entendaient sans le voir, Pierre Schaeffer développe le concept d'acousmatique en démontrant tout l'apport de la vue à l'audition. Voir Pierre Schaeffer, Traité des objets musicaux: essai interdisciplines, Paris, Éditions du Seuil, coll. «Pierres vives », 1977, p. 91 sq. 
Ce débat, qui n'est pas clos ainsi que nous allons le voir, visait la préservation d'une relation «en direct» - live ${ }^{12}$ - qu'on n'allait pas tarder à ériger en valeur distinctive ${ }^{13}$, essentielle et transcendante du théâtre autour du concept de présence ${ }^{14}$. C'était évidemment nouveau.

Dans son imposant Dictionnaire historique et pittoresque du théâtre et des arts qui s'y rattachent publié en 1885 , qui est l'un des premiers ouvrages encyclopédiques du théâtre, Arthur Pougin examine méthodiquement le champ sémantique du mot «théâtre » dont il relève plus d'une demi-douzaine d'acceptions. Aucune d'elles ne traite de la présence, qu'il s'agisse de celle de l'acteur ou de celle du spectateur. Pougin consacre également un long paragraphe à l'«art théâtral » :

Voici un mot dont la portée est vaste, un mot qui représente et sous-entend un ensemble de qualités très diverses, dont l'heureuse réunion peut seule permettre d'atteindre la perfection toute relative permise à la nature humaine. L'art théâtral est un art particulièrement complexe, très varié dans ses manifestations comme dans ses moyens, parlant tout ensemble à l'esprit, à l'imagination, à l'oreille et aux yeux, et

12. Ce terme usuel dans le monde du spectacle de scène, de la radio et de la télévision combine les propriétés de présence, de direct et de ce que, à l'époque du numérique, on qualifie de «temps réel». Il s'oppose, en principe, au différé, au retransmis, à l'enregistré. Mais les technologies numériques ont pour effet de brouiller ces catégories dont la pertinence s'amenuise.

13. En référence au critère de distinction de Pierre Bourdieu: «La dialectique de la prétention et de la distinction qui est au principe des transformations du champ de production se retrouve dans l'espace des consommations: elle caractérise ce que j'appelle la lutte de concurrence». Pierre Bourdieu, Questions de sociologie, Paris, Les Éditions de Minuit, coll. «Documents», 1984, p. 201.

14. Ce concept n'est pas facile à cerner, d'abord parce qu'il est complexe, ensuite parce qu'il n'a pas cessé d'évoluer. Je m'en tiendrai, pour les besoins de cet article, à la définition qu'en propose Philip Auslander: «In theatrical parlance, presence usually refers either to the relationship between actor and audience - the actor as manifestation before an audience-or, more specifically, to the actor's psychophysical attractiveness to the audience, a concept related to that of charisma. Concepts of presence are grounded in notions of actorly representation; the actor's presence is often thought to derive from her embodiment of, or even possession by, the character defined in a play text, from the (re)presentation of self through the mediation of character, or, in the Artaudian/Grotowskian/Beckian line of thought, from the archetypal psychic impulses accessible through the actor's physicality». Voir Philip Auslander, Presence and Resistance: Postmodernism and Cultural Politics in Contemporary American Performance, Ann Arbor, University of Michigan Press, coll. «Theater: Theory/Text/Performance», 1993, p. 37. 
produisant par cela même des impressions d'une rare puissance et d'une étonnante intensité ${ }^{15}$.

Si on peut voir dans cette définition un écho du projet globalisant de Wagner ${ }^{16}$ ou du rêve synesthésique des symbolistes, on n'y trouve pas la moindre trace de cette notion de présence. Et pour cause! Comme le rappelle Philip Auslander, les Grecs de l'Antiquité allaient au théâtre sans savoir ni se demander si c'était du théâtre live $e^{17}$ parce qu'il n'existait pas d'autres formes de théâtre. De la même façon, le concept de présence n'a pu émerger que quand des formes médiatisées de représentation sont apparues.

Il y a évidemment des enjeux ontologiques à toute innovation technologique - ou tout transfert intermédiatique (d’un média à un média) - enjeux qui deviennent majeurs et donc terriblement inquiétants lorsque l'innovation - ou le transfert - a la capacité de bouleverser les protocoles en usage et les valeurs établies. C'était le cas à l'époque électrique, ce l'est tout autant, sinon plus, à l'époque actuelle. Les technologies fondées sur le numérique sont souples et ont un potentiel de transformation nettement supérieur à celui des technologies électriques. Il n'est donc pas étonnant que les inquiétudes suscitées par la médiation électrique soient non seulement relayées mais qu’elles soient amplifiées dans le discours contemporain.

\section{LE CORPS DE L'ACTEUR ET LA PRÉSENCE COMME FONDEMENTS IDENTITAIRES}

Dans la section de L'ouvre d'art à l'époque de sa reproductibilité qu'il consacre au théâtre, Walter Benjamin fait très largement écho aux critiques et appréhensions qu'exprime le dramaturge et metteur en scène Luigi Pirandello à l'égard

15. Arthur Pougin, Dictionnaire historique et pittoresque du théâtre et des arts qui s'y rattachent: poétique, musique, danse, pantomime, décor, costume, machinerie, acrobatisme, Paris, Librairie De Firmin-Didot, 1885. Entrée «théâtre», p. 704-705; entrée «Art théâtral », p. 63.

16. L’influence du concept de Gesamtkunstwerk défendu par Wagner et perpétué par les symbolistes contemporains de Pougin.

17. «It was the development of recording technologies that made it possible to perceive existing representations as live. Prior to the advent of those technologies (e.g., sound recording and motion pictures), there was no such thing as live performance, for that category has meaning only in relation to an opposing possibility », Philip Auslander, Liveness: Performance in a Mediatized Culture, Londres et New York, Routledge, 2008, p. 56 . 
du cinéma dans son roman Si gira ${ }^{18}$. Choisissant un opérateur de vues animées comme protagoniste, Pirandello témoigne dans cet ouvrage du peu de considération, pour ne pas dire du mépris, qu'il a pour le «nouveau divertissement». Il voit dans le cinéma ni plus ni moins que la fin de l'art de l'acteur. Le cinéma comme anti-théâtre!

Les acteurs de cinéma [...] se sentent comme en exil. En exil non seulement de la scène, mais encore d'eux-mêmes. Ils remarquent confusément, avec une sensation de dépit, d'indéfinissable vide et même de faillite, que leur corps est presque subtilisé, supprimé, privé de sa réalité, de sa vie, de sa voix, du bruit qu'il produit en se remuant $[\ldots]^{19}$.

\section{Ou encore,}

C'est l'acteur de théâtre en personne qui présente au public sa performance artistique à l'état définitif; celle de l'acteur de cinéma réclame la médiation de tout un appareillage. [...] [L]'interprète du film, ne présentant pas lui-même sa performance au public, n'a pas, comme l'acteur de théâtre, la possibilité d'adapter son jeu, en cours de représentation, aux réactions des spectateurs²o.

La présence immédiate de l'acteur au spectateur et leur interaction, auxquelles Pougin n'avait fait aucune allusion quarante ans plus tôt, s'imposent désormais comme LA qualité distinctive du spectacle théâtral et semblent bien garantir sa supériorité ontologique face aux performances «reproduites ». L'effet dépréciatif s'étend non seulement aux médias électriques mais à toutes les formes de médiations réalisées à l'aide de dispositifs technologiques - qu'ils soient acoustiques ou électriques.

On pourrait sans doute reprocher à Benjamin d'avoir accordé une bien trop large place - en fait, toute la place - au Pirandello de ces années 1910 et de ne s'en être tenu qu'à lui. Si on peut présumer que l'opinion de Pirandello reflétait

18. Le texte original (italien) est publié en 1915. La version française, On tourne, date de 1925. Voir Luigi Pirandello, On tourne, Paris, Éditions du Sagittaire, coll. « de la revue européenne », 1925 .

19. Walter Benjamin, «L'œuvre d'art à l'époque de sa reproductibilité technique », version de 1939, dans (Euvres, vol. 3, trad. Maurice de Gandillac, Rainer Rochlitz et Pierre Rusch, Paris, Gallimard, coll. «Folio Essais», 2000, p. 291. Les citations de Benjamin sont tirées de Léon Pierre-Quint, «Signification du cinéma », dans Léon Pierre-Quint, Germaine Dulac, Lionel Landry, Abel Gance (dir.), L'art cinématographique, II, Paris, F. Alcan, 1927, p. 14 sq.

2o. Walter Benjamin, «L'œuvre d'art à l'époque de sa reproductibilité technique», CEuvres, vol. 3, p. 289. 
effectivement un sentiment assez partagé dans le milieu théâtral de l'époque, elle n'exprimait ni un point de vue unanime ni un point de vue progressiste. En ces années d'avant-guerre, Gabriele D’Annunzio, le grand rival de Pirandello, et les futuristes ne cessaient de vanter, quant à eux, les vertus et le potentiel du cinéma. D'ailleurs Pirandello lui-même n’allait pas tarder à se ranger de leur côté, effectuant une véritable conversion au cinéma ${ }^{21}$, pour reprendre le mot ironique de Fabio Andreazza.

On comprend le parti pris de Benjamin. Ce concept de présence, si cher à l'épistémè théâtrale contemporaine, doit en effet être compris comme l'application à la réalité théâtrale du principe du hic et nunc de l'œuvre d'art qu'il a développé dans cet essai22 majeur, ce hic et nunc qui fait son « unicité » et « constitue ce qu'on appelle son authenticité23». En cette époque où le monde du théâtre entreprend de construire son discours identitaire ${ }^{24}$, tout écart par rapport à cette «authenticité » serait donc perçu négativement. Si les « conditions nouvelles dans lesquelles le produit de la reproduction technique peut être placé ne remettent peut-être pas en cause l'existence même de l'œuvre d'art, elles déprécient en tout cas son hic et nunc ${ }^{25}$ ». Les défenseurs du théâtre traditionnel ont fait leur ce modèle dualiste où le «reproduit» - ce qui inclut le médiatisé - accuse inévitablement une perte par rapport à l'original. Et ils présentent le spectacle théâtral comme l'un des ultimes lieux de résistance à la domination de la reproductibilité technique. Cette thèse bien discutable est, en l'occurrence et paradoxalement, appuyée par Benjamin lui-même.

21. En particulier quand il entrevoit la possibilité d'adapter sa pièce Six personnages en quête d'auteur à l'écran. Voir à ce propos l'article de Fabio Andreazza «La conversion de Pirandello au cinéma », Actes de la recherche en sciences sociales, nº 161-162, "Cinéma et intellectuels », mars 2006, p. 32-41.

22. Il a aussi abordé ce principe dans ses essais «Petite histoire de la photographie », Euvres, vol. 2, p. 295-321, et «Sur quelques motifs baudelairiens », Euvres, vol. 3, p. 329-390.

23. Walter Benjamin, «L'œuvre d'art à l'époque de sa reproductibilité technique », Euvres, vol. 3, p. 274 .

24. Cette quête identitaire n'est pas spécifique au théâtre comme l'attestent les essais retentissants de Jean-Paul Sartre, Qu'est-ce que la littérature?, Paris, Gallimard, coll. «Folio Essais », 1993 et André Bazin, Qu'est-ce que le cinéma?, Paris, Éditions du Cerf, coll. « $7^{\mathrm{e}}$ art », 1999 .

25. Walter Benjamin, «L'œuvre d'art à l'époque de sa reproductibilité technique», CEuvres, vol. 3, p. 275. 
Rien, en effet, ne s'oppose plus radicalement à l'œuvre d'art entièrement envahie par la reproduction technique, voire, comme dans le film, née de cette reproduction, que le théâtre.

Benjamin n'a pas seulement reconnu le statut particulier du théâtre à l'heure de la reproductibilité, il a souligné la fonction essentielle qu'y joue l'acteur. C'est à lui que le théâtre doit sa singularité, voire sa supériorité. «Le dernier progrès du film consiste à réduire l'acteur à un accessoire, qu'on choisit caractéristique [...] et qu'on situe à la bonne place ${ }^{26}$. »

L'interférence «dénaturante » de la caméra dont se plaignait Pirandello ${ }^{27}$ est la même qui motivait les accusations de «trahison » proférées à l'encontre des technologies de reproduction sonore qu'évoque Jonathan Sterne. On aurait pu croire que trois quarts de siècle plus tard, la réalité de la pratique - qui, elle, ne se formalisait pas de l'invasion des technologies de reproduction et des transferts intermédiatiques - aurait relégué au rang d’argument historique classé ce réquisitoire empreint de nostalgie, mais il n'en est rien. Dans un ouvrage marquant publié en 1993, Peggy Phelan rappelait avec insistance cette caractéristique qu'elle présente comme un fait ontologique primordial pour le théâtre:

only life is in the present. Performance cannot be saved, recorded, documented, or otherwise participate in the circulation of representations of representations: once it does so, it becomes something other than performance.

Cette affirmation est suivie d'un avertissement contre toute contamination médiatique de la scène qui n'est pas sans rappeler les accusations relevées par Sterne ou la «faillite» dénoncée par Pirandello.

To the degree that performance attempts to enter the economy of reproduction, it betrays and lessens the promise of its own ontology ${ }^{28}$.

Ainsi, frayer avec «l'économie de la reproduction» au milieu des années 1990, alors que déferlait la vague numérique, c’était encore trahir la «promesse ontologique » du théâtre!

26. Walter Benjamin, «L'œuvre d'art à l'époque de sa reproductibilité technique », CEuvres, vol. 3, p. 291-292.

27. Sa réflexion portait sur le cinéma muet.

28. Peggy Phelan, Unmarked: The Politics of Performance, Londres et New York, Routledge, 1993, p. 146. 
Dans son essai sur la mémoire, l'histoire et l'oubli²9 publié la même année que l'ouvrage de Bolter et Grusin ${ }^{\circ}$, Paul Ricœur, approfondit sa réflexion sur la construction identitaire et ses enjeux amorcée une dizaine d'années plus tôt Reprenant les concepts de «mêmeté » et d'«ipséité », cette dernière correspondant davantage aux arguments des défenseurs - dont Phelan - d'une sauvegarde d'une pureté originelle de la pratique, Ricoeur souligne les failles du processus d'affirmation identitaire et les dérives qu'il peut provoquer. Car l'identité n'est pas sûre! "Qu'est-ce qui fait la fragilité de l'identité? Eh bien, c'est le caractère purement présumé, allégué, prétendu de l'identité.32» Et c'est justement pour légitimer cette présomption-allégation-prétention que les défenseurs de l'identité - en l'occurrence, de l'ipséité - en appellent avec tant d'insistance au passé.

Le cœur du problème, c'est la mobilisation de la mémoire au service de la quête, de la requête, de la revendication d'identité.

Il faut nommer comme première cause de la fragilité de l'identité son rapport difficile au temps; difficulté primaire qui justifie précisément le recours à la mémoire, en tant que composante temporelle de l'identité, en conjonction avec l'évaluation du présent et la projection du futur ${ }^{33}$.

On comprend que Ricœur évite, dans ces pages, d'aborder la question de l'essentialisme mais comment qualifier autrement le discours virulent qui, de Pirandello à Phelan, prône la supériorité ontologique du théâtre sur toutes les pratiques de reproduction et défend ses qualités singulières (ou présentées comme telles) 34 ?

29. Paul Ricœur, La mémoire, l'histoire, l'oubli, Paris, Éditions du Seuil, coll. «L'ordre philosophique », 2000.

30. Jay David Bolter et Richard Grusin, Remediation: Understanding New Media.

31. Dans Paul Ricœur, Soi-même comme un autre, Paris, Éditions du Seuil, coll. «L'ordre philosophique», 1990.

32. Paul Ricœur, La mémoire, l'histoire, l'oubli, p. 98.

33. Paul Ricœur, La mémoire, l'histoire, l'oubli, p. $9^{8}$.

34. Opposé à l'existentialisme, au nominalisme, au constructionnisme, l'essentialisme doit être traité avec prudence surtout dans le champ des sciences humaines et des pratiques artistiques. Je l'emploie aussi dans son sens le plus large: certaines propriétés d'une pratique sont - prétendues - permanentes, inaltérables et transhistoriques. 
Identifiant comme première cause de la fragilité de l'identité le rapport au temps - à la mémoire -, Ricœur aborde ensuite les effets de la présence de l'altérité dont le surgissement semble inévitablement marqué de violence.

Deuxième cause de fragilité, la confrontation avec autrui, ressentie comme une menace. C'est un fait que l'autre, parce que autre, vient à être perçu comme un danger pour l'identité propre $[\ldots]$.

Troisième cause de fragilité, l'héritage de la violence fondatrice. C'est un fait qu'il n'existe pas de communauté historique qui ne soit née d'un rapport qu'on peut dire originel à la guerre ${ }^{35}$.

Fondé sur une identité forgée et légitimée par la mémoire, mais alimentée par une actualité perçue ou présentée comme désespérante - pour ne pas dire «violente» -, le discours essentialiste s'est imposé jusqu’à devenir hégémonique. Et pour cause! D'un point de vue de « résistant», comment comprendre l'avènement ininterrompu pendant tout un siècle de médias nouveaux et de nouvelles pratiques autrement que comme une érosion du statut - de dominant - du théâtre et un appauvrissement de ses qualités?

En raison de la primauté qu'elle accorde aux relations, l'approche intermédiale est évidemment peu préoccupée par les questions d'identité et de territoire - et donc de frontières -, mais c'est justement son intérêt pour les mouvements complexes et omnidirectionnels à l'œuvre dans le champ des médias et des technologies, sa capacité d'en mesurer l'ampleur et d'en saisir l'impact qui permettent de mieux comprendre les tenants et aboutissants du discours essentialiste, dans sa nature comme dans sa conjoncture.

\section{L'APPROCHE HISTORIENNE}

Ce discours essentialiste qui se développe et s'impose pendant près d'un siècle - si l'on tient compte du rejet initial des technologies de reproduction sonore dès les années 1880 - trouve des alliés dans tous les camps, y compris celui des historiens. Profondément marqués par la pensée sémioticienne qui domine le champ des études théâtrales pendant le dernier quart du $20^{\circ}$ siècle, les historiens ont relayé et légitimé le discours identitaire autour du concept de théâtralité puis de performativité. Or il allait vite apparaître que la théâtralité 
n'est pas propre au théâtre et qu'une position théâtrocentrique qui situerait la scène comme matrice unique de la théâtralité est difficilement défendable.

Loin de réduire l'importance du réflexe identitaire et du discours quasi hégémonique qu'il a produit, l'histoire intermédiale en propose au contraire une lecture en trois temps fondés sur des moments-clés de l'histoire des médias au $20^{\text {e }}$ siècle. Selon ce que propose Peter Boenisch ${ }^{6}{ }^{6}$, l'évolution du théâtre depuis un siècle est le produit direct des vagues technologiques successives qui ont déferlé sur les pratiques médiatiques et des réactions - en ressac - qu’elles y ont provoquées. L'histoire du théâtre, de la modernité à la période actuelle, est une histoire médiatique.

On ne s'étonnera pas que le premier cas relevé par Boenisch survienne au moment de la révolution électrique et de la naissance du cinéma. Optant assez rapidement pour l'illusionnisme ${ }^{37}$, le cinéma en serait arrivé à concurrencer le théâtre sur son propre terrain et l'aurait condamné à se redéfinir en changeant son rapport au réel. La théâtralisation de la scène correspondrait donc d'abord et avant tout, selon Boenisch, à une volonté de se distinguer et se distancer du cinéma: à faire ce que le cinéma ne pouvait pas faire. Il y a là plus qu'un paradoxe. En affirmant une identité fondée sur la théâtralité, le théâtre reniait sa propre et longue quête réaliste et sacrifiait la scène à l'italienne - elle n'a pas disparu pour autant! - qui visait justement à occulter sa médialité. Au cinéma qui cherchait la transparence ${ }^{38}$ pour rapprocher le spectateur du réel représenté à l'écran puis à travers les haut-parleurs, le théâtre réagissait en affichant sa matérialité, son «hypermédiacie».

Boenisch élargit ainsi la formule-choc de l'historien Thomas Postlewait «theatricalism as anti-realism » à «theatricalism as anti-realism as anti-cinema ${ }^{39}$ ». L'équation est sans doute rapide et soulève une question de fond: est-ce que le vaste et long mouvement de "théâtralisation » du théâtre qui va des avantgardes - le constructivisme, l'expressionnisme, en particulier - au théâtre

36. Peter Boenisch, «Aesthetic Art to Aisthetic Act: Theatre, Media, Intermedial Performance », dans Freda Chapple et Chiel Kattenbelt (dir.), Intermediality in Theatre and Performance, Amsterdam et New York, Rodopi, 2006, p. 103-115.

37. Les premières vues animées étaient des documentaires.

38. Ce qui correspond à l' «immediacy» de Bolter et Grusin. Voir Jay David Bolter et Richard Grusin, Remediation: Understanding New Media.

39. « $[\mathrm{M}]$ odernist theatre reconfigured another aspect of theatricality according to the realist-theatricalist polarity, whereby realist conventions sought to erase the apparent operations of theatricalism ", dans Tracy C. Davis, Thomas Postlewait (dir.), Theatricality, Cambridge et New York, Cambridge University Press, 2003, p. 11. 
épique de Bertolt Brecht ne serait que l'effet d'une stratégie de repositionnement médiatique? La «coïncidence » relevée par Boenisch vaut d'être approfondie ${ }^{40}$.

Le deuxième moment de résistance du théâtre à une remédiation dangereuse - ou perçue comme telle - se produit, toujours selon le système de périodisation de Boenisch, lors du développement de la télévision - puis de la vidéo. La crise débute dans les années 1950. Dans un mode envahi par l'artifice, le théâtre s'impose par son authenticité, il est l'ultime « residue of pure and authentic culture in a world of mass-media and television daftness ${ }^{41} »$. Dominée par la quête d'une communication qu'on veut «vraie», intense et qui, on le comprend, ne saurait être médiatisée par quelque technologie que ce soit, une nouvelle génération de créateurs entreprend de dépouiller la scène de tous ses artifices.

Alors que le studio de télévision et le plateau de tournage se présentent comme des bazars technologiques au service du factice, la scène est réduite à l'essentiel - un plateau rudimentaire, un éclairage minimal, un espace public sommaire - pour ne laisser place qu'aux acteurs communiant directement avec leurs spectateurs. C'est ce partage de l'émotion pure, palpable, physique, de sens à sens, dénuée de toute interférence technologique qui guide autant la recherche de Jerzy Grotowski que celle de Peter Brook. C'est l'ère du «théâtre pauvre », de «l'espace vide»; c'est celle aussi du «théâtre direct», ramené à sa plus simple expression - son «esthétique économique » comme l'appelait JeanClaude Germain ${ }^{42}$ - instauré par la création collective dans sa période la plus turbulente (de 1968 à 1975). Le vide contre l'abondance, l'art comme «forme d'expérience maximale» contre l'art qui « renforçait la structure » - ainsi que le déplorait Julian Beck, cofondateur du Living Theatre ${ }^{43}$ - la vérité profonde contre le mirage du matérialisme, le corps de l'acteur - mis à nu, naturel, « entier» - contre sa reproduction, la présentation contre la re-présentation.

40. Et une approche sociomédiale révélerait le rôle moteur du «milieu» dans cette conjoncture.

41. Tracy C. Davis, Thomas Postlewait (dir.), Theatricality, p. 103.

42. Jean-Claude Germain est directeur et fondateur du Théâtre du Même Nom, l'une des troupes de création collective les plus marquantes du Québec à cette époque (voir Jane Baldwin, Jean-Marc Larrue et Christiane Page [dir.], Vies et morts de la création collective, Boston, Vox Theatri, 2008). Le «théâtre pauvre» que prône Grotowski est perçu comme une force. Voir Julian Beck, La vie du théâtre, trad. Fanette et Albert Vander, Paris, Gallimard NRF, coll. «Pratique du théâtre ", 1978, p. 43.

43. Julian Beck revenait sur l'expérience de cette troupe phare du mouvement des collectifs au festival d'Avignon, en 1968, année où, dit-il, «la culture est morte». Voir Julian Beck, La vie du théâtre, p. 214. 
Boenisch voit dans cette période un autre moment fort de la pensée essentialiste. Je suggère plutôt qu'elle en marque le déclin irréversible. Rappelons-le, les années 1970, qui voient se multiplier les méga-spectacles, sont aussi celles de la performance et du triomphe de la postmodernité. Or, dans leur entreprise de déconstruction, les artisans de la postmodernité théâtrale se sont d'abord et avant tout attaqués à la présence et au type de relation - jugée factice par eux - qu'elle induit entre spectateur et acteur. En rejetant la présence, les praticiens de la scène postmoderne se sont attaqués aux hiérarchies établies et aux manipulations qu'elles permettaient. La présence, rappelle la performeuse canadienne Vera Frenkel, tient du charisme qui déclenche une projection «by which we attribute to others, especially a leader, entertainer or artist the secret images within ourselves ${ }^{44}$ ». Elle poursuit en affirmant que la performance postmoderne doit échapper au piège de la présence en évitant ces projections mutuelles grâce à «a non charismatic understanding which permits us not to believe so readily in the other as the keeper of our treasure and our disease ${ }^{45}$ ». Cet argument sert de fondement à l'essai Presence and Resistance que Philip Auslander consacre à la performance en 1994. La démarche des créateurs postmodernes, appuyée par les théoriciens de la postmodernité, doit être comprise en réaction contre le théâtre des années 1960-1970 et non en continuité avec lui.

Postmodernist theater and performance artists and theorists implicitly and explicitly rejected the premises of the ecstatic political theater of the 1960s: its reliance on the presence of the performer $[\ldots]^{46}$.

Philip Auslander poursuit son entreprise «déconstructionniste» dans un autre essai percutant publié cinq ans plus tard, Liveness: Performance in a Mediatized Culture ${ }^{47}$. Il y mène une charge dévastatrice contre ce concept fondateur que la critique avait jusque-là étonnamment épargné. Et cela survient alors que le théâtre subit l'effet de la révolution numérique qui transforme autant ses

44. Vera Frenkel, «Discontinuous Notes on and after a Meeting of Critics, by One of the Artists Present», Artscanada, n 240-241, 1981, p. 37-38 (cité par Philip Auslander, Presence and Resistance-Postmodernism and Cultural Politics in Contemporary American Performance, Ann Arbor, The University of Michigan Press, 1994, p. 43).

45. Vera Frenkel, «Discontinuous Notes on and after a Meeting of Critics, by One of the Artists Present», p. 43.

46. Philip Auslander, Presence and Resistance: Postmodernism and Cultural Politics in Contemporary American Performance, p. 42.

47. Publié une première fois à New York par Routledge en 1999. 
modes de représentation que ses processus créatifs et narratifs ${ }^{4}$. L'ouvrage d'Auslander et l'âpre débat qu' il suscite constituent, selon Boenisch, le troisième temps de l'histoire médiatique du théâtre.

Auslander démontre que la notion de présence n'est pas propre au théâtre et qu'elle ne correspond même pas à la réalité de la scène telle que nous la vivons depuis un siècle. Les arguments sont forts et ont eu l'effet d'un électrochoc dans le champ des études théâtrales. Non seulement les défenseurs du théâtre s'étaient-ils convaincus eux-mêmes de la supériorité ontologique de la présence «vivante» sur toutes les formes de reproduction - donc du théâtre sur les médias tels la radio et le cinéma —, mais ils en auraient convaincu les praticiens des autres médias. En érigeant cette caractéristique en vertu cardinale exclusive à la scène, ils suscitaient toutes les convoitises, dont celle de la télévision naissante.

Il y a en effet deux aspects importants au discours essentialiste. Obsédé par la question de la définition de l'identité, il construit non seulement cette identité en dévalorisant les autres - au moins dans un premier temps -, mais il rend ces autres redevables de la pratique qu'il défend. Il s'est dégagé de cela, pendant près d'un siècle, l'image euphorisante d'un théâtre servant de matrice aux médias plus récents. Ceux-ci, à commencer par le cinéma des premiers temps, ne se seraient pas privés. Ils auraient débauché le public et les agents du théâtre, ils se seraient approprié son organisation institutionnelle, ses stratégies narratives, ses modalités communicationnelles (la frontalité, l'expérience commune), ses espaces. Exprimant un sentiment largement partagé chez les théoriciens et les historiens traditionnels du théâtre, Jack Poggi n’hésite pas à assimiler le processus à du pillage.

Early cinema took over and reformed a theatrical vocabulary and also rapidly usurped the theatre's cultural position as the dominant form of entertainment. Indeed, film had thoroughly routed the theatre by 1926 , so there was little left to pillage when television arrived in force some twenty years later ${ }^{49}$.

Mais Poggi oubliait «l'essentiel»! Préservé de ce pillage, le concept de présence si attaché au mode de représentation scénique allait séduire les pionniers de la télévision et devenir leur principal argument, non pas à l'encontre d'un théâtre dépecé et qui semblait à l'agonie, mais à l'encontre du cinéma, qui

48. C'est le sujet d'un autre essai marquant publié par Janet Horowitz Murray, Hamlet on the Holodeck. The Future of Narrative in Cyberspace, New York, The Free Press, 1997.

49. Jack Poggi, Theater in America: The Impact of Economic Forces, 1870-1967, Ithaca, Cornell University Press, 1968, p. 85-86, cité par Philip Auslander, Liveness: Performance in a Mediatized Culture, p. 12. 
reposait, lui aussi, sur le dispositif filmographique. La chose est moins étonnante que ne le suggère Auslander.

There can be no question that the advent of film had a devastating cultural-economic impact on the theatre, but that fact, taken by itself, leaves an important question unanswered. If the theatre as a popular form had been so thoroughly usurped by film in the 1920s that it was hardly even a force to be reckoned with when television came around, why [souligné par l'auteur] did television embrace «the theatre as a model for representation» $[\ldots]$ as the cinema itself had done in its earliest days, rather than model itself on film? As a camera-bound medium, television might well have striven to be cinematic; but instead it strove to be theatrical5․

Dans un marché hautement concurrentiel, il était en effet inévitable que le concept de présence, irrécupérable par le cinéma, serve, en vertu de ses valeurs d'authenticité et d'unicité - le hic et nunc de Benjamin - de stratégie d'émergence et de légitimation pour la radio puis pour la télévision, prouvant bien l'hégémonie du discours essentialiste au-delà de la sphère théâtrale.

\section{DISCOURS ET PRAXIS: DE L'APRÈS-AUSLANDER AU TEMPS DE L'INTERMÉDIALITÉ}

La charge d'Auslander n'a pas seulement ébranlé un discours tenace, elle a permis de révéler le gouffre qui le séparait de la réalité de la pratique, et cela bien avant la vague de la performance postmoderne. Pendant des décennies, le discours essentialiste a en effet ignoré, occulté ou minimisé les innombrables apports des autres médias et des technologies au théâtre et à ses agents depuis l'avènement de l'électricité, à commencer par l'éclairage électrique. Les mouvements de va-et-vient étaient et sont pourtant continuels et le théâtre n'a pas cessé au cours du dernier siècle d'importer, d'emprunter, d'exploiter tout ce qui pouvait enrichir et faciliter ses créations, ainsi qu'il l'avait toujours fait! Et rien ne permet de dire que le théâtre ait été le continuel perdant de ces flux et reflux provoqués par les diverses innovations technologiques et révolutions médiatiques. Rappelons quelques faits. Les artistes et gestionnaires de théâtre ont été les principaux responsables et bénéficiaires du succès initial du gramophone et de la radio; le théâtre a très vite compris le potentiel du téléphone nouvellement créé et s'en est servi sans tarder pour augmenter sa diffusion «en direct» hors de ses salles ${ }^{51}$; la bande-son, qui fait son entrée dans les salles de théâtre au cours des

50. Philip Auslander, Liveness: Performance in a Mediatized Culture, p. 12.

51. C'est le cas du Théâtrophone, dont on trouve des variantes dans la plupart des capitales à la fin du $19^{\mathrm{e}}$ siècle, dans lequel on peut voir une première tentative de système de radiodiffusion... théâtrale. 
années 1950 et qui est une composante indispensable de la théâtralité contemporaine, provient du cinéma et a été implantée au théâtre par des réalisateurs de film ${ }^{2}$. Que dire encore de tous les recours aux projections d'images fixes et animées, qui jalonnent l'histoire des genres théâtraux, du théâtre épique aux genres populaires du théâtre victorien, de la revue au mélodrame et à la féerie, depuis l'invention du projecteur à lampe?

Langoisse du changement, qui selon Auslander, «underlies many performance theorists' desire to reassert the integrity of the live and the corrupt, co-opted nature of the mediatized ${ }^{3} 3$ ", a non seulement rendu suspecte, dans le discours essentialiste, toute forme d'assistance technologique à l'acteur - à l'exception notoire de l'éclairage électrique - , elle a également dévié l'attention des analystes d'autres dangers.

L'art - indéfini et changeant - de l'acteur sur lequel se fonde l'art du théâtre a en effet ses détracteurs au sein même de la pratique, mais ceux-là - Meyerhold, Craig, Maeterlinck (qui allait jusqu'à souhaiter un théâtre libéré de ses acteurs en chair et en os - un théâtre véritablement post-humain) -, en dépit des transformations radicales qu'ils préconisaient ou qu'ils ont réalisées (dans le cas de Meyerhold), ont été relativement épargnés par les tenants de ce discours montrant à quel point il se nourrissait d'abord et aveuglément de «technophobie ».

Or, créateurs et spectateurs ont adopté sans réticence - et même parfois avec empressement - les technologies numériques qui envahissent désormais nos scènes et nos lieux de production et de création, comme elles envahissent notre espace public et privé. Elles s'y trouvent visiblement en terrain hospitalier. On comprend que, dans ce contexte, le concept de présence perde de sa pertinence, sapant les fondements mêmes du discours identitaire. Lacteur d'aujourd'hui, peut, en effet, être présent en temps réel mais non dans l'espace, il peut être présent dans l'espace mais occulté ou supplanté par sa propre image médiatisée, il peut donner l'illusion d'être là mais ne pas y être - sans que personne ne s'en rende compte. L'acteur peut se fragmenter, se multiplier; être là olfactivement mais non visuellement ou inversement, se disjoncter (associer une voix à un autre corps), etc. Bref, on peut difficilement prétendre aujourd'hui que la présence et le direct sont la nécessité ultime, le dernier rempart qui peut, hors de tout doute, caractériser la pratique théâtrale et fixer son identitést.

52. Le réalisateur Luchino Visconti a été l'un des premiers à utiliser une bande-son sur scène.

53. Philip Auslander, Liveness: Performance in a Mediatized Culture, p. 44.

54. Auslander conclut que le tribunal serait le seul système où le live existe vraiment... 
Les analystes de la représentation ont saisi toute la portée de ce changement, délaissant récemment la quête de la présence pour celle de ses «effets » - «effets de présence» - et François Jost, en réexaminant la stratégie d'émergence et de développement de la télévision, en venait à se demander en 2006 «[à] qui profite la question de l'identité5s? » La prochaine interrogation pourrait bien porter sur sa raison d'être: à quoi sert-elle? À quoi a-t-elle servi? Et on voit bien que ce sujet si lié aux relations intermédiales concerne aussi les socialités. Mais nous n’en sommes pas tout à fait là. Parmi ces « effets de présence » plane toujours la tentation essentialiste. Aujourd'hui encore, affirmer que le théâtre est un média et que l'acteur relève de sa matérialité suscite l'embarras. Assimiler ce dernier au concept d'«appareil» développé par Jean-Louis Déotte $5^{6}$ ou le considérer comme un «média technique» - tel que l'affirme Lars Elleström7 - heurte les sensibilités.

Les études intermédiales n'ont évidemment pas ignoré les innombrables créations qui, depuis La biche au bois ${ }^{58}$, misent sur les ressources et la présence sur scène d'autres médias ou de technologies nouvelles, mais ce n'est qu'en 2006 que de tels spectacles et les questions qu'ils soulèvent ont été abordés selon une approche intermédiale. L'ouvrage Intermediality in Theatre and Performance ${ }^{59}$, publié sous la direction de Freda Chapple et Chiel Kattenbelt, marquait ainsi une première percée majeure de l'intermédialité auprès des théoriciens du théâtre et confirmait tout autant la nécessité que l'urgence de revoir le fonctionnement du théâtre et son histoire.

55. C'est le titre même de son article: «À qui profite la question de l'identité? », MédiaMorphoses, $\mathrm{n}^{\circ}$ 16, avril 2006, p. 55-59.

56. Voir en particulier Jean-Louis Déotte, Marion Froger et Silvestra Mariniello (dir.), Appareil et intermédialité, Paris, L'Harmattan, coll. «Esthétiques», 2007.

57. "A technical medium is any object or body that "realizes," "mediates," or "displays" basic and qualified media. A technical medium can thus be described as "form," while basic and qualified media are "content," and it can be identified according to the range of basic media it has the capacity of mediating. » Lars Elleström, «The Modalities of Media: A Model for Understanding Intermedial Relations ", Lars Elleström (dir.), Media Borders, Multimodality, and Intermediality, Basingstoke, Palgrave Macmillan, 2010.

58. Présentée au Théâtre du Châtelet de Paris à partir du 14 novembre 1896, cette féerie comportait la projection d'une vue animée en couleurs réalisée à l'aide d'un chronophotographe Demenÿ-Gaumont.

59. Freda Chapple et Chiel Kattenbelt (dir.), Intermediality in Theatre and Performance. 